\title{
A MATHEMATICAL REVISION OF THE LANDAU-LIFSHITZ EQUATION
}

Chein-Shan Liu

Department of Civil Engineering, National Taiwan University, Taipei, Taiwan, R.O.C.

Kuo-Ching Chen

Institute of Applied Mechanics, National Taiwan University, Taipei, Taiwan, R.O.C., kcc@spring.iam.ntu.edu.tw

Chau-Shioung Yeh

Department of Civil Engineering, National Taiwan University, Taipei, Taiwan, R.O.C.

Follow this and additional works at: https://jmstt.ntou.edu.tw/journal

Part of the Civil and Environmental Engineering Commons

\section{Recommended Citation}

Liu, Chein-Shan; Chen, Kuo-Ching; and Yeh, Chau-Shioung (2009) "A MATHEMATICAL REVISION OF THE LANDAULIFSHITZ EQUATION," Journal of Marine Science and Technology. Vol. 17: Iss. 3, Article 8.

DOI: $10.51400 / 2709-6998.1959$

Available at: https://jmstt.ntou.edu.tw/journal/vol17/iss3/8

This Research Article is brought to you for free and open access by Journal of Marine Science and Technology. It has been accepted for inclusion in Journal of Marine Science and Technology by an authorized editor of Journal of Marine Science and Technology. 


\section{A MATHEMATICAL REVISION OF THE LANDAU-LIFSHITZ EQUATION}

\section{Acknowledgements}

The financial supports provided by the National Science Council for CS under the Grant NSC 93-2212-E-019-008 and for KC under NSC 97-2221-E-002-12 are gratefully acknowledged. 


\title{
A MATHEMATICAL REVISION OF THE LANDAU-LIFSHITZ EQUATION
}

\author{
Chein-Shan Liu*, Kuo-Ching Chen**, and Chau-Shioung Yeh*
}

Key words: magnetization, Landau-Lifshitz equation, Lie-Poisson system, two-generator, double-bracket flow, isospectral flow, coadjoint orbit, rotation group, Lorentz group.

\begin{abstract}
For the Landau-Lifshitz equation a Lie type linear system representation in the Minkowski space $\mathbb{M}^{3+1}$ has been derived previously [25]. The internal symmetry group is a proper orthochronous Lorentz group $\mathrm{SO}_{o}(3,1)$, and the numerical method based on the internal symmetry was developed in [29]. This paper derives another four new representations of the Landau-Lifshitz equation. We prove that this equation admits two generators: one conservative and one dissipative, as well as two brackets: Poisson bracket and dissipative bracket. Upon embedding the Landau-Lifshitz equation into a skew-symmetric matrix space, we can develop a double-bracket flow representation. The conserved magnetization magnitude is just the result of the isospectrality for an isospectral flow equation. Finally, on the cotangent bundle of an invariant manifold of the constant magnetization magnitude, we introduce the Lie-Poisson bracket to construct an evolutional differential equations system. The magnetization trajectory traces a coadjoint orbit in the Poisson manifold under a coadjoint action of the rotation group $S O(3)$. The six different representations including the one by Bloch et al. [3] are compared.
\end{abstract}

\section{INTRODUCTION}

The hysteretic phenomenon of ferromagnetic materials has attracted great scholarly attention in the last century. Many models have been proposed to describe the micro-magnetic dynamics of ferromagnetic media. Among them, the Landau-Lifshitz model [20] has been widely used, in which the following equation plays a central role:

Author for correspondence: Kuo-Ching Chen (e-mail: kcc@spring.iam.ntu.edu.tw). *Department of Civil Engineering, National Taiwan University, Taipei, Taiwan, R.O.C.

**Institute of Applied Mechanics, National Taiwan University, Taipei, Taiwan, R.O.C.

$$
\dot{\mathbf{M}}=-\gamma \mathbf{M} \times \mathbf{H}_{\text {eff }}-\frac{\gamma \alpha}{M_{s}} \mathbf{M} \times\left(\mathbf{M} \times \mathbf{H}_{\text {eff }}\right) .
$$

From the above equation it is apparent that $\mathbf{M} \cdot \dot{\mathbf{M}}=0$, and consequently the magnitude of magnetization vector $\mathbf{M}(t)$ is conserved, i.e., $\|\mathbf{M}(t)\|=M_{s}=$ constant. Throughout this paper, a superimposed dot denotes the time derivative, a dot between two vectors stands for their scalar product, and $\|\bullet\|$ presents the magnitude of a vector. The two material parameters $\gamma>0$ and $\alpha \geq 0$ are the absolute value of gyromagnetic ratio and the Gilbert damping constant [14], respectively. The effective field $\mathbf{H}_{\text {eff }}$ is the sum of the applied field, the demagnetizing field, the anisotropy field, and the exchange field.

The Landau-Lifshitz equation is essential to the interpretation of the dynamics of domain wall [41], ferromagnetic resonance [11], and magnetization switching in thin film recording media [38], and its extension beyond the restriction that this equation is introduced for small magnetization motions and for the case of high magnetic symmetry with an isotropic damping parameter has been made by Safonov [36] and Safonov and Bertram [37]. Recently, an exact analytical result was obtained for a magnetic body exhibiting rotational symmetry about a certain axis and the external field being circularly polarized in the perpendicular plane [2]. However, exact solutions for the nonlinear magnetization problems are yet to be explored. Usually, the majority of nonlinear studies are carried out by the numerical integration techniques [12, $19,27,28,39]$.

It will be useful to recall some of the basic and essentially well known elements of generalized Hamiltonian formulation of mechanical systems. The classical Hamiltonian mechanics is endowed with an even-dimensional phase space. In practice, there are many mechanical systems whose phase spaces are not canonical $[6,7,26]$. That is, the phase manifold does not admit a cotangent bundle structure on it, but still has a Poisson bracket equipped with the properties of skew-symmetry, bilinearity, the Leibniz identity and the Jacobi identity. The most famous example is the Euler equations, governing the motion of rigid body.

Suppose that $P$ is a manifold. If there is a bracket $\{\bullet, \bullet\}$ defined on the function space $\mathbb{C}(P)$, which possesses the following properties: 
Skew-Symmetry: $\{F, G\}=-\{G, F\}$,

Bilinearity:

$$
\{\lambda F+\mu G, H\}=\lambda\{F, H\}+\mu\{G, H\}, \lambda, \mu \in \mathbb{R},
$$

Jacobi identity:

$$
\{F,\{G, H\}\}+\{G,\{H, F\}\}+\{H,\{F, G\}\}=0,
$$

Leibniz identity: $\{F G, H\}=F\{G, H\}+\{F, H\} G$,

then $(P,\{\bullet, \bullet\})$ is a Poisson manifold [30]. If an observable function $F: P \mapsto \mathbb{R}$ of a dynamical system can be governed by a generalized Hamiltonian function $H$ through

$$
\dot{F}=\{F, H\}
$$

then $(P,\{\bullet, \bullet\}, H)$ is called a generalized Hamiltonian system.

Let $H: P \mapsto \mathbb{R}$ be a smooth function on $P$. The generalized Hamiltonian vector field $\mathbf{X}_{H}$ associated with $H$ is a unique smooth vector field on $P$, which for every smooth function $F: P \mapsto \mathbb{R}$ satisfies

$$
\mathbf{X}_{H}(F)=\{F, H\}
$$

Instead of the non-degeneracy of classical Poisson bracket, the bracket defined on the non-canonical Poisson manifold is permitted degenerate.

Suppose that $C: P \mapsto \mathbb{R}$ is a non-constant smooth function on $P$. If $\{C, F\}=0$ for all smooth function $F: P \rightarrow \mathbb{R}$, then $C$ is a Casimir function on $P$. When $P$ is a finite-dimensional manifold with dimensions $n$, the local coordinates of $P$ can be assigned as $\mathbf{x}=\left(x_{1}, \ldots, x_{n}\right)$, and the Poisson bracket on $P$ can be written as

$$
\{F, G\}:=J_{i j} \frac{\partial F}{\partial x_{i}} \frac{\partial G}{\partial x_{j}},
$$

where $J_{i j}(\mathbf{x})$ is a Poisson tensor. In this paper the Einstein summation convention is adopted for repeated indices.

Given an $n \times n$ matrix function $\mathbf{J}(\mathbf{x})=J_{i j}(\mathbf{x})$ defined on the open set $P \subset \mathbb{R}^{n}$, the necessary and sufficient conditions of $\mathbf{J}(\mathbf{x})$ to be a Poisson tensor are

$$
\begin{gathered}
J_{i j}=-J_{j i}, i, j=1,2, \cdots, n, \\
J_{i \ell} J_{j k, \ell}+J_{j \ell} J_{k i, \ell}+J_{k \ell} J_{i j, \ell}=0, \quad i, j, k=1,2, \cdots, n,
\end{gathered}
$$

where $J_{j k, \ell}$ denotes $\partial J_{j k} / \partial x_{\ell}$.

For all smooth function $H: P \mapsto \mathbb{R}$ defined on $P$, the bundle mapping $B: T^{*} P \mapsto T P$ is denoted by $B(d H(\mathbf{x}))=\mathbf{X}_{H} \mid \mathbf{x}$. $T P$ and $T^{*} P$ are, respectively, the tangent and cotangent bundles on the Poisson manifold $P$. The rank of the Poisson bracket at a point $\mathbf{x} \in P$ is defined as the rank of the linear mapping $\left.B\right|_{\mathbf{x}}: T_{\mathbf{x}}^{*} P \mapsto T_{\mathbf{x}} P$. A point $\mathbf{x}$ on the Poisson manifold $P$ is called a regular point, if the ranks for all points in the neighborhood of $\mathbf{x} \in P$ are the same; otherwise, $\mathbf{x}$ is a singular point. The rank of $B$ at $\mathbf{x} \in P$ and the rank of Poisson tensor $\mathbf{J}(\mathbf{x})$ at point $\mathbf{x}$ are the same. Because of the skew-symmetry of $\mathbf{J}(\mathbf{x})$ the rank is always even.

Suppose that the rank of Poisson tensor $\mathbf{J}(\mathbf{x})$ at a regular point $\mathbf{x}_{0}$ is $n-m, m>0$, then there are $m$ functionally independent Casimir functions defined in the neighborhood of the point $\mathbf{x}_{0}$.

Especially, when $J_{i j}(\mathbf{x})$ is a linear function of $\mathbf{x}$, the bracket (8) is called a Lie-Poisson bracket, and (6) is a Lie-Poisson system written as

$$
\dot{\mathbf{x}}=\mathbf{J}(\mathbf{x}) \nabla H(\mathbf{x}),
$$

where the gradient operator $\nabla$ denotes the derivative with respect to $\mathbf{x}$. We usually write such $J_{i j}(\mathbf{x})$ to be

$$
J_{i j}=C_{i j}^{k} x_{k}
$$

where $C_{i j}^{k}=-C_{j i}^{k}$ and the Jacobi identity (10) takes the form:

$$
C_{i j}^{\ell} C_{\ell k}^{r}+C_{j k}^{\ell} C_{\ell i}^{r}+C_{k i}^{\ell} C_{\ell j}^{r}=0
$$

It is known that for this case the underlying space can be given a Lie algebra structure with the structure constants $C_{i j}^{k}$ in a suitable basis [30].

The Lie-Poisson system is naturally formulated in the dual space of a Lie algebra $\mathcal{G}$. The solutions of the system are coadjoint orbits of a certain Lie group, constrained in the nonlinear submanifolds of $\mathcal{G}^{*}$ known as the symplectic foliations. In recent years, the applications that fit into the Lie-Poisson formalism and the extensions to dissipative systems are numerous [3, 35]. Also, for its important applications in the physical systems there were some integrators developed to preserve the Lie-Poisson structure [5, 10, 13, 21, 29, 31].

For the undamped case of (1)

$$
\dot{\mathbf{M}}=-\gamma \mathbf{M} \times \mathbf{H}_{\text {eff }},
$$

Bloch et al. [3] have considered a Poisson bracket of the rigid body type,

$$
\{F, G\}(\mathbf{M})=\mathbf{M} \cdot[\nabla F(\mathbf{M}) \times \nabla G(\mathbf{M})],
$$

such that by introducing the generator

$$
S=\gamma \mathbf{M} \cdot \mathbf{H}_{\text {eff }},
$$

one can obtain

$$
\dot{M}_{i}=\left\{M_{i}, S\right\}=\varepsilon_{i j k} M_{k}(\nabla S)_{j},
$$


where $\varepsilon_{i j k}$ is the permutation symbol. It can be seen that $J_{i j}=$ $\varepsilon_{i j k} M_{k}$ is a Lie-Poisson tensor, and (17) is a Lie-Poisson system. However, under this formulation there has a weak point that $S$ is not an invariance of the system unless $\mathbf{H}_{\text {eff }}$ is a constant vector. Then, Bloch et al. [3] extended their formulation to system (1) by adding a symmetric dissipative bracket:

$$
\{\{F, G\}\}(\mathbf{M})=\frac{\alpha}{M_{s}}[\mathbf{M} \times \nabla F(\mathbf{M})] \cdot[\mathbf{M} \times \nabla G(\mathbf{M})],
$$

and obtained a two-bracket form of system (1):

$$
\dot{M}_{i}=\left\{M_{i}, S\right\}+\left\{\left\{M_{i}, S\right\}\right\} .
$$

Previously, Liu [22, 24] has developed a Jordan algebra basis of the Landau-Lifshitz equation, and then Liu [25] has derived a Lie type linear system representation in the Minkowski space. Here, we analyze the Landau-Lifshitz equation from another four new different aspects, namely, a twogenerator formalism, an isospectral flow, a double-bracket form, and a Lie-Poisson bracket formulation which rendering the coadjoint orbit on the invariant manifold of the system with a constant $H=\alpha\|\mathbf{M}\|^{2} /\left(2 M_{s}^{2}\right)$ rather than the above $S=$ $\gamma \mathbf{M} \cdot \mathbf{H}_{\text {eff }}$. This paper not only manifests the mathematically structural elegance and the diversity in the guise of the Landau-Lifshitz equation, but also provides a new thought that this nonlinear dissipative equation can be examined from different aspects. The significance of these four representations is that these different forms are associated with their related mathematical physics so that the intrinsic properties of the Landau-Lifshitz equation, which had not yet been found before, are thoroughly uncovered.

\section{TWO-GENERATOR FORMALISM}

Corresponding to the one-generator formulation as shown in equation (19) for the Landau-Lifshitz equation, there is a two-generator formulation. The two-generator bracket formalism is the one that appears first in the original development for the dynamics of dissipative systems by Kaufman [18], Morrison [32] and Grmela [15]. Recent progress of this formalism leads to the GENERIC (general equation for the nonequilibrium reversible-irreversible coupling) framework $[1,16,34]$, in which the time evolution of any isolated thermodynamic system can be written in the form

$$
\frac{d \mathbf{x}}{d t}=\mathbb{L}(\mathbf{x}) \frac{\delta E(\mathbf{x})}{\delta \mathbf{x}}+\mathbb{M}(\mathbf{x}) \frac{\delta S(\mathbf{x})}{\delta \mathbf{x}},
$$

where $\mathbb{L}$ and $\mathbb{M}$ are certain operators, $\delta \bullet / \delta \mathbf{x}$ signifies a Fréchet derivative, and $E$ and $S$ represent, respectively, the total energy and entropy expressed in terms of the state variables $\mathbf{x}$. The use of two generators, the energy for the reversible dynamics and the entropy for the irreversible dynamics, is the charac- teristic feature of GENERIC. The major advantage of the GENERIC framework is that it can be derived from Hamilton's equations for classical systems or from Heisenberg's equations for quantum systems. Two notable equations that can be derived in the GENERIC context are the Navier-Stokes equation [9] and the Boltzmann equation [33]. Rewriting the Landau-Lifshitz equation in the context of the two-generator formalism is the purpose of this section.

Let us define a unit vector

$$
\mathbf{m}:=\frac{\mathbf{M}}{\|\mathbf{M}\|}=\frac{\mathbf{M}}{M_{S}},
$$

and for saving notations we use a new time scale $\gamma M_{S} t$ still denoted by $t$ and a new field $\mathbf{H}_{\text {eff }} / M_{S}$ denoted by $\mathbf{H}$, such that (1) can be rearranged to

$$
\dot{\mathbf{m}}=\hat{\mathbf{H}} \mathbf{m}+\alpha \mathbf{H}-\alpha \mathbf{H} \cdot \mathbf{m m},
$$

where

$$
\hat{\mathbf{H}}:=\left[\begin{array}{ccc}
0 & -H_{3} & H_{2} \\
H_{3} & 0 & -H_{1} \\
-H_{2} & H_{1} & 0
\end{array}\right]
$$

is skew-symmetric, a superimposed dot presents the derivative with respect to the new time $t$, and $H_{i}, i=1,2,3$, are three independent components of $\mathbf{H}$. The field $\mathbf{H}$ can be treated as an input on (22) and in general is a function of $\mathbf{m}$. Now we take an assumption as also adopted by Bloch et al. [3] that $\mathbf{H}$ is a constant vector. Under such restriction the elegance of the mathematical structure of the Landau-Lifshitz equation in the two-generator formalism can be manifested.

Theorem 1. The Landau-Lifshitz equation (22), admitting two generators: one conservative $E=\|\mathbf{m}\|^{2} / 2$ and one dissipative $S=\mathbf{H} \cdot \mathbf{m}$, can be written as

$$
\dot{\mathbf{m}}=\hat{\mathbf{H}} \nabla E+\boldsymbol{\eta} \nabla S,
$$

where $\hat{\mathbf{H}}=-\hat{\mathbf{H}}^{T}$ and

$$
\boldsymbol{\eta}:=\alpha\left(\mathbf{I}_{3}-\mathbf{m} \otimes \mathbf{m}\right)
$$

are, respectively, a skew-symmetric structural tensor and a non-negative metric tensor. Furthermore, there hold two degenerate conditions

$$
\begin{aligned}
\hat{\mathbf{H}} \nabla S & =0, \\
\boldsymbol{\eta} \nabla E & =0 .
\end{aligned}
$$

Proof. In order to obtain a two-generator formulation of the Landau-Lifshitz equation (22), let us rewrite it with the following form:

$$
\dot{\mathbf{m}}=\hat{\mathbf{H}} \nabla E+\alpha\left[\mathbf{I}_{3}-\mathbf{m} \otimes \mathbf{m}\right] \mathbf{H},
$$


Table 1. Comparisons of six representations of the Landau-Lifshitz equation.

\begin{tabular}{|c|c|c|c|c|c|c|}
\hline Equation & Space & Bracket(s) & Generator(s) & Invariance & Lie algebra & Lie group \\
\hline$(19)$ & $\mathbb{E}^{3}$ & Lie-Poisson + Dissipative & One & Coadjoint & - & - \\
\hline$(24)$ & $\mathbb{E}^{3}$ & Poisson + Dissipative & Two & Casimir & - & - \\
\hline$(44)$ & Skew(3) & Lie + Double & - & Eigenvalue & $s o(3)$ & $S O(3)$ \\
\hline$(47)$ & $S k e w(3)$ & Double & - & Eigenvalue & $s o(3)$ & $S O(3)$ \\
\hline$(55)$ & $\mathbb{M}^{3+1}$ & - & Cone & $s o(3,1)$ & $S O_{o}(3,1)$ \\
\hline$(67)$ & $\mathcal{G}^{*}$ & Lie-Poisson & One & Coadjoint & $\mathbf{M}_{i}^{*} \in \operatorname{So}(3)$ & $S O(3)$ \\
\hline
\end{tabular}

where $\otimes$ denotes the dyadic product, and

$$
E(\mathbf{m})=\frac{\|\mathbf{m}\|^{2}}{2}
$$

is a conservative quantity preserved by the Landau-Lifshitz equation as demonstrated above. For the later use we may call $\|\mathbf{m}\|=1$ an invariant manifold of the system, i.e., $\mathbf{m} \in \mathbb{S}^{2}$. Hence, $\nabla E=\mathbf{m}$ follows directly and (28) is verified by noting $\mathbf{m} \otimes \mathbf{m H}=\mathbf{H} \cdot \mathbf{m m}$.

Let

$$
S(\mathbf{m})=\mathbf{H} \cdot \mathbf{m}
$$

be the quantity dominated the dissipation of the system. $\nabla S=$ $\mathbf{H}$ follows directly and (24) is thus verified in view of (25) and (28).

Now, we prove that $\boldsymbol{\eta}$ is non-negative definite. From (25) the symmetry of $\boldsymbol{\eta}$ is obvious. Then, by

$$
\mathbf{v}^{\mathrm{T}}\left[\mathbf{I}_{3}-\mathbf{m} \otimes \mathbf{m}\right] \mathbf{v}=\|\mathbf{v}\|^{2}-(\mathbf{v} \cdot \mathbf{m})^{2} \geq 0, \quad \forall \mathbf{v} \in \mathbb{R}^{3},
$$

we prove that $\alpha\left(\mathbf{I}_{3}-\mathbf{m} \otimes \mathbf{m}\right) \geq 0$ and thus $\boldsymbol{\eta}$ is non-negative definite. In above the superscript "T" denotes the transpose. In addition, the two degenerate conditions (26) and (27) are easily identified with the help of (23), (25), (29) and (30). $\square$

For arbitrary two functions $F(\mathbf{m})$ and $G(\mathbf{m})$, let us define the Poisson bracket by

$$
\begin{aligned}
\{F, G\}(\mathbf{m}): & =\nabla F(\mathbf{m}) \cdot[\hat{\mathbf{H}} \nabla G(\mathbf{m})] \\
& =-\mathbf{H} \cdot[\nabla F(\mathbf{m}) \times \nabla G(\mathbf{m})],
\end{aligned}
$$

and the dissipative bracket by

$$
\begin{aligned}
\{\{F, G\}\}(\mathbf{m}): & =\nabla F(\mathbf{m}) \cdot[\mathbf{\eta}(\mathbf{m}) \nabla G(\mathbf{m})] \\
& =\alpha[\mathbf{m} \times \nabla F(\mathbf{m})] \cdot[\mathbf{m} \times \nabla G(\mathbf{m})] .
\end{aligned}
$$

Since $\hat{\mathbf{H}}$ is skew-symmetric and is assumed to be independent of $\mathbf{m}$, it satisfying (9) and (10) is a Poisson tensor. The skewsymmetric $\hat{\mathbf{H}}$ and the non-negative definite $\boldsymbol{\eta}$ guarantee the skew-symmetry of the Poisson bracket defined in (32) and the symmetry of the dissipative bracket defined in (33). Then, a bracket, called the two-generator bracket, composed of the above two brackets is the underlying bracket structure for (24). The governing equation for this system is thus simply given by

$$
\dot{F}=\{F, E\}+\{\{F, S\}\}=\nabla F \cdot(\hat{\mathbf{H}} \nabla E)+\nabla F \cdot(\boldsymbol{\eta} \nabla S) .
$$

As the consequences of (23) and (25)-(27) we have

$$
\begin{gathered}
\dot{E}=\{E, E\}+\{\{E, S\}\}=\nabla E \cdot(\hat{\mathbf{H}} \nabla E)+\nabla E \cdot(\boldsymbol{\eta} \nabla S)=0, \\
\dot{S}=\{S, E\}+\{\{S, S\}\}=\nabla S \cdot(\hat{\mathbf{H}} \nabla E)+\nabla S \cdot(\boldsymbol{\eta} \nabla S) \geq 0,
\end{gathered}
$$

which indicate that $E$ is a conservative quantity and $S$ has a natural physical interpretation as the entropy of this system. Especially, because of

$$
\{\{E, F\}\}=0
$$

for arbitrary differentiable function $F(\mathbf{m}), E$ may be viewed as a Casimir function of the dissipative bracket dynamics.

It deserves to note that our formulation of the LandauLifshitz equation is different from that proposed by Bloch et al. [3], which as presented in (19) is an one-generator formulation. The comparisons of these two formulations and the others to be discussed below are summarized in Table 1.

\section{ISOSPECTRAL FLOW AND DOUBLE-BRACKET FLOW}

The double-bracket flow

$$
\dot{\mathbf{Y}}=[[\mathbf{Y}, \mathbf{N}], \mathbf{Y}], t \geq 0, \mathbf{Y}(0)=\mathbf{Y}_{0} \in \operatorname{Sym}(n)
$$

was introduced simultaneously by Brockett [4] and Chu and Driessel [8]. In (38), $\operatorname{Sym}(n)$ denotes the set of all real $n \times n$ symmetric matrices, $\mathbf{N}(t) \in \operatorname{Sym}(n)$ is a given matrix function, and $[\bullet, \bullet]$ is the usual matrix Lie bracket. The most important feature of (38) is that it is a special case of the isospectral flow equation [17]: 


$$
\dot{\mathbf{Y}}=[\mathbf{B}(t, \mathbf{Y}), \mathbf{Y}], t \geq 0, \mathbf{Y}(0)=\mathbf{Y}_{0} \in \operatorname{Sym}(n)
$$

where $\mathbf{B}: \mathbb{R}^{+} \times \operatorname{Sym}(n) \mapsto s o(n)$, and the set $s o(n)$ is a Lie algebra composed of all $n \times n$ real skew-symmetric matrices. From (39) it follows a solution:

$$
\mathbf{Y}(t)=\mathbf{Q}(t) \mathbf{Y}_{0} \mathbf{Q}^{\mathrm{T}}(t), t \geq 0,
$$

where $\mathbf{Q}$ is a solution of

$$
\dot{\mathbf{Q}}=\mathbf{B}\left(t, \mathbf{Q} \mathbf{Y}_{0} \mathbf{Q}^{\mathrm{T}}\right) \mathbf{Q}, t \geq 0, \mathbf{Q}(0)=\mathbf{I}_{n} .
$$

Since it is a Lie-group equation, $\mathbf{Q}$ evolves in $S O(n)$, and therefore (40) presents a similar transformation of the initial value $\mathbf{Y}_{0}$ onto $\mathbf{Y}(t)$. In other words, the eigenvalues of $\mathbf{Y}$ in (39) (and hence in (38)) are invariances of the flow equation, not varying with $t$.

An extension of (38) can be made by allowing $\mathbf{Y}$ to be skew-symmetric. In order to derive the isospectral flow and double-bracket flow formalisms for the Landau-Lifshitz equation, let us return to the $3 \times 3$ skew-symmetric real matrix as the one already shown in (23) for the case $\mathbf{H}$. For each $\mathbf{x} \in \mathbb{R}^{3}$ we can assign a $3 \times 3$ skew-symmetric matrix to represent it:

$$
\hat{x} \mapsto \mathbf{x} \mapsto \hat{\mathbf{x}}:=\left[\begin{array}{ccc}
0 & -x_{3} & x_{2} \\
x_{3} & 0 & -x_{1} \\
-x_{2} & x_{1} & 0
\end{array}\right]
$$

Suppose that $\mathbf{x}, \mathbf{y} \in \mathbb{R}^{3}$ and that $\hat{\mathbf{x}}$ and $\hat{\mathbf{y}}$ are the corresponding $3 \times 3$ skew-symmetric matrices, then through some derivations we can identify the following relation:

$$
\mathbf{x} \times \mathbf{y}=\hat{\mathbf{x}} \mathbf{y} \equiv \hat{\mathbf{x}} \hat{\mathbf{y}}-\hat{\mathbf{y}} \hat{\mathbf{x}}=[\hat{\mathbf{x}}, \hat{\mathbf{y}}]
$$

where $\times$ is the usual cross product of three-dimensional vectors and $[\bullet, \bullet]$ is the matrix Lie bracket of three-dimensional matrices. Equation (43) is a key point for further development, and under the above sense we can assign an isospectral flow for (1).

Theorem 2. The Landau-Lifshitz equation. (22) admits an isospectral flow representation:

$$
\dot{\hat{\mathbf{m}}}=[\hat{\mathbf{H}}, \hat{\mathbf{m}}]+[[\hat{\mathbf{m}}, \alpha \hat{\mathbf{H}}], \hat{\mathbf{m}}],
$$

with $t \geq 0$ and $\hat{\mathbf{m}}(0)=\hat{\mathbf{m}}_{0} \in \operatorname{Skew}(3)$, where Skew(3) denotes the set of all real $3 \times 3$ skew-symmetric matrices. The eigenvalues of $\hat{\mathbf{m}}$ are 0 and $\pm i\|\mathbf{m}\|$, the latter two of which are preserved by the flow equation.

Proof. Simplifying (1) by the new time scale and the new vector field $\mathbf{H}$ as that demonstrated below (21) and by using (43), it is not difficult to derive (44), which can be further written as

$$
\dot{\hat{\mathbf{m}}}=[\hat{\mathbf{H}}+[\hat{\mathbf{m}}, \alpha \hat{\mathbf{H}}], \hat{\mathbf{m}}] .
$$

Since $\hat{\mathbf{H}}+[\hat{\mathbf{m}}, \alpha \hat{\mathbf{H}}]$ is skew-symmetric, Eq. (44) is indeed an isospectral flow equation. The preservation of $\|\mathbf{m}\|$ is a direct result of (44).

It deserves to note that the first and second terms on the right-hand side of (44) correspond, respectively, to the first and second terms on the right-hand side of (24). The two brackets in (44) are respectively the Lie bracket and the double bracket in a literal sense.

In the corotated frame $\mathbf{m}$ is given as $\mathbf{m}^{\mathrm{R}}=\mathbf{R}^{\mathrm{T}} \mathbf{m}$ and $\mathbf{H}$ as $\mathbf{H}^{\mathrm{R}}=\mathbf{R}^{\mathrm{T}} \mathbf{H}$, where the rotation matrix $\mathbf{R}$ satisfies

$$
\dot{\mathbf{R}}=\hat{\mathbf{H}} \mathbf{R}, \mathbf{R}(0)=\mathbf{I}_{3} .
$$

Then, from (22) we can prove the following result.

Theorem 3. In the corotated frame, the Landau-Lifshitz equation (22) admits a double-bracket flow representation:

$$
\dot{\hat{\mathbf{m}}}^{R}=\left[\left[\hat{\mathbf{m}}^{R}, \alpha \hat{\mathbf{H}}^{R}\right], \hat{\mathbf{m}}^{R}\right],
$$

with $t \geq 0$ and $\hat{\mathbf{m}}^{R}(0)=\hat{\mathbf{m}}_{0} \in \operatorname{Skew}(3)$.

Proof. From (22) we have

$$
\dot{\mathbf{m}}^{\mathrm{R}}=\alpha \mathbf{H}^{\mathrm{R}}-\alpha \mathbf{H}^{\mathrm{R}} \cdot \mathbf{m}^{\mathrm{R}} \mathbf{m}^{\mathrm{R}}
$$

due to $\mathbf{R}^{\mathrm{T}} \mathbf{R}=\mathbf{I}_{3}$. The above equation can be written as

$$
\dot{\mathbf{m}}^{\mathrm{R}}=\alpha\left(\mathbf{m}^{\mathrm{R}} \times \mathbf{H}^{\mathrm{R}}\right) \times \mathbf{m}^{\mathrm{R}}
$$

due to the invariance of $\mathbf{m}^{\mathrm{R}} \cdot \mathbf{m}^{\mathrm{R}}=\mathbf{m} \cdot \mathbf{m}=1$. Then, skewsymmetrizing the above equation and employing the equivalence as specified in (43) we can arrive at (47).

\section{LINEAR REPRESENTATION IN THE MINKOWSKI SPACE}

The above techniques employed to derive the bracket formulas for the Landau-Lifshitz equation rely on the introduction of flow equations in different spaces. These derivations prompt us to define an integrating factor for (22),

$$
X^{0}:=\exp \left(\alpha \int_{0}^{t} S(\xi) d \xi\right)=\exp \left(\alpha \int_{0}^{t}[\mathbf{H}(\xi) \cdot \mathbf{m}(\xi)] d \xi\right) .
$$

The above equation together with (30) assert that

$$
X^{0}\left(t_{1}\right) \geq X^{0}\left(t_{2}\right), t_{1} \geq t_{2},
$$

provided that $S(t) \geq 0$, which implies that the angle between the magnetization and the effective field is less than $90^{\circ}$. Then (51) indicates that $X^{0}$ is a time-like variable of the system, and is pointing to the future. 
Accordingly, we can prove the following theorem with a slight extension of the results given by Liu [25].

Theorem 4. The Landau-Lifshitz equation (22) admits a linear system $\dot{\mathbf{X}}=\mathbf{A X}$ representation in the four-dimensional Minkowski space with $\mathbf{X} \in \mathbb{M}^{3+1}$, which holds the cone condition $\mathbf{X}^{\mathrm{T}} \mathbf{g X}=0$, where $\mathbf{g}$ is a Minkowski metric with signature 2. Suppose that $\mathbf{X}_{1}(t)$ and $\mathbf{X}_{2}(t)$ are two solutions of $\dot{\mathbf{X}}=\mathbf{A X}$, then $\mathbf{X}_{1}^{\mathrm{T}}(t) \mathbf{g} \mathbf{X}_{2}(t)$ is an invariance.

Proof. Upon taking advantage of the integrating factor defined in $(50)$, we can rearrange $(22)$ to

$$
\frac{d}{d t}\left(X^{0} \mathbf{m}\right)=X^{0} \hat{\mathbf{H}} \mathbf{m}+\alpha X^{0} \mathbf{H}
$$

On the other hand, from (50) it follows directly that

$$
\dot{X}^{0}=\alpha X^{0} \mathbf{H} \cdot \mathbf{m} .
$$

Let us introduce the augmented state vector:

$$
\mathbf{X}=\left[\begin{array}{l}
\mathbf{X}^{s} \\
X^{0}
\end{array}\right]=\left[\begin{array}{l}
X^{1} \\
X^{2} \\
X^{3} \\
X^{0}
\end{array}\right]:=X^{0}\left[\begin{array}{c}
\mathbf{m} \\
1
\end{array}\right],
$$

and then (52) and (53) are combined to

$$
\dot{\mathbf{X}}=\mathbf{A X}
$$

where

$$
\mathbf{A}:=\left[\begin{array}{cc}
\hat{\mathbf{H}} & \alpha \mathbf{H} \\
\alpha \mathbf{H}^{\mathrm{T}} & 0
\end{array}\right] .
$$

The model originally formulated for $\mathbf{m} \in \mathbb{S}^{2}$ can be transformed into a model in the augmented state space of $\mathbf{X}$ with a cone condition [23]:

$$
\mathbf{X}^{\mathrm{T}} \mathbf{g X}=\left(X^{0}\right)^{2}\left[\|\mathbf{m}\|^{2}-1\right]=0
$$

in terms of the Minkowski metric $\mathbf{g}$,

$$
\mathbf{g}=\left[\begin{array}{cc}
\mathbf{I}_{3} & \mathbf{0}_{3 \times 1} \\
\mathbf{0}_{1 \times 3} & -1
\end{array}\right] .
$$

The vector space of augmented state $\mathbf{X}$ endowed with the Minkowski metric tensor $\mathbf{g}$ is referred to as a Minkowski space designated as $\mathbb{M}^{3+1}$.

For the system (55) let us consider the following two equations:

$$
\dot{\mathbf{X}}_{1}=\mathbf{A} \mathbf{X}_{1}
$$

$$
\dot{\mathbf{X}}_{2}=\mathbf{A} \mathbf{X}_{2}
$$

which under the same $\mathbf{A}$ but with different initial conditions $\mathbf{X}_{1}(0)$ and $\mathbf{X}_{2}(0)$; hence, the two solutions $\mathbf{X}_{1}$ and $\mathbf{X}_{2}$ are not fully identical in the range of $t \geq 0$. Applying $\mathbf{X}_{2}^{\mathrm{T}} \mathbf{g}$ to the first equation and then integrating the resultant we have

$$
\begin{aligned}
\int_{0}^{t} \mathbf{X}_{2}^{\mathrm{T}}(\xi) \mathbf{g} \dot{\mathbf{X}}_{1}(\xi) d \xi & =\int_{0}^{t} \mathbf{X}_{2}^{\mathrm{T}}(\xi) \mathbf{g A}(\xi) \mathbf{X}_{1}(\xi) d \xi \\
& =-\int_{0}^{t} \mathbf{X}_{2}^{\mathrm{T}}(\xi) \mathbf{A}^{\mathrm{T}}(\xi) \mathbf{g} \mathbf{X}_{1}(\xi) d \xi \\
& =-\int_{0}^{t} \mathbf{X}_{1}^{\mathrm{T}}(\xi) \mathbf{g A}(\xi) \mathbf{X}_{2}(\xi) d \xi \\
& =-\int_{0}^{t} \mathbf{X}_{1}^{\mathrm{T}}(\xi) \mathbf{g} \dot{\mathbf{X}}_{2}(\xi) d \xi
\end{aligned}
$$

in which we have used the Lie algebra property of $\mathbf{A}$ :

$$
\mathbf{A}^{\mathrm{T}} \mathbf{g}+\mathbf{g A}=0
$$

On the other hand, integrating by part we obtain

$$
\int_{0}^{t} \mathbf{X}_{2}^{\mathrm{T}}(\xi) \mathbf{g} \dot{\mathbf{X}}_{1}(\xi) d \xi=\left.\mathbf{X}_{2}^{\mathrm{T}} \mathbf{g} \mathbf{X}_{1}\right|_{0} ^{t}-\int_{0}^{t} \mathbf{X}_{1}^{\mathrm{T}}(\xi) \mathbf{g} \dot{\mathbf{X}}_{2}(\xi) d \xi
$$

Comparing (61) and (63) leads to

$$
\mathbf{X}_{2}^{\mathrm{T}}(t) \mathbf{g} \mathbf{X}_{1}(t)=\mathbf{X}_{2}^{\mathrm{T}}(0) \mathbf{g} \mathbf{X}_{1}(0)
$$

which means that $\mathbf{X}_{1}^{\mathrm{T}} \mathbf{g} \mathbf{X}_{2}=\mathbf{X}_{2}^{\mathrm{T}} \mathbf{g} \mathbf{X}_{1}$ is an invariance of the system.

The theorem manifests that a state of $\mathbf{m}$ on the sphere $\|\mathbf{m}\|=1$ corresponds to an augmented state $\mathbf{X}$ on the right circular cone $\left\{\mathbf{X} \mid \mathbf{X}^{\mathrm{T}} \mathbf{g X}=0\right\}$ emanating from $\mathbf{X}=0$ of the Minkowski space. In addition, as a consequence of (64) we can prove that

$$
\mathbf{m}_{1}(t) \cdot \mathbf{m}_{2}(t)=1+\frac{\mathbf{m}_{1}(0) \cdot \mathbf{m}_{2}(0)-1}{X_{1}^{0}(t) X_{2}^{0}(t)}
$$

when insert (54) for $\mathbf{X}$ and (58) for $\mathbf{g}$ into (64). Since $S$ is not a decreasing function of $t$ as shown in (36), both $X_{1}^{0}$ and $X_{2}^{0}$ defined by (50) tend to infinite. Therefore, from (65) it follows that

$$
\lim _{t \rightarrow \infty} \mathbf{m}_{1}(t) \cdot \mathbf{m}_{2}(t)=1 \text {. }
$$

When taking $\left\|\mathbf{m}_{1}(t)\right\|=\left\|\mathbf{m}_{2}(t)\right\|=1$ into account, the above equation means that the magnetization orientations align to the same direction independent on the initial orientations.

Because $\mathbf{A}$ is an element of the real Lie algebra $s o(3,1)$ as shown in (62), the generated transformation $\mathbf{G}$ corresponding to $\mathbf{A}$ is an element of the proper orthochronous Lorentz group $S O_{o}(3,1)$. Liu [25] has employed the above symmetry into a 
numerical solution of the Landau-Lifshitz equation, and found that the new numerical method is stable, robust, accurate and preserving the manifold invariant.

\section{LIE-POISSON BRACKET FORMULATION}

For the undamped case, i.e., $\alpha=0$, of the Landau-Lifshitz equation we have mentioned in Section 1 that Bloch et al. [3] have given a Lie-Poisson system description by viewing $S$ as a generator $[12,14]$. However, for the most applications in micromagnetics of the damped Landau-Lifshitz equation there does not yet have a Lie-Poisson system description. In this section we construct a Lie-Poisson bracket formulation of the Landau-Lifshitz equation, stressing the coadjoint orbit invariant behavior of this equation.

Equation (48) can be written as

$$
\dot{\mathbf{m}}^{R}=\mathbf{J} \nabla H,
$$

where

$$
H=\frac{\alpha}{2}\left\|\mathbf{m}^{\mathrm{R}}\right\|^{2}=\frac{\alpha}{2}\|\mathbf{m}\|^{2}
$$

is an invariant function of the system as a generalized Hamiltonian function, and

$$
\mathbf{J}=\mathbf{H}^{\mathrm{R}} \otimes \mathbf{m}^{\mathrm{R}}-\mathbf{m}^{\mathrm{R}} \otimes \mathbf{H}^{\mathrm{R}}
$$

is a Poisson tensor. Then we can prove the following results.

Theorem 5. The Landau-Lifshitz equation (22) is a LiePoisson system. The solutions of (22) are the coadjoint orbits of the Lie group $\mathrm{SO}(3)$, constrained in the invariant manifold of $\mathcal{G}^{*}$ as a symplectic foliation with the generalized Hamiltonian function $H$ constant on it. Even the Poisson tensor $\mathbf{J}$ is degenerate, there exists no Casimir function of this system.

Proof. We can prove that $\mathbf{J}$ satisfies (9) and (10). The first condition of skew-symmetry is obvious. Let us write

$$
\begin{gathered}
J_{i j}=H_{i}^{\mathrm{R}} m_{j}^{\mathrm{R}}-m_{i}^{\mathrm{R}} H_{j}^{\mathrm{R}}, \\
J_{i j, \ell}=H_{i}^{\mathrm{R}} \delta_{j \ell}-\delta_{i \ell} H_{j}^{\mathrm{R}} .
\end{gathered}
$$

By using them we have

$$
\begin{aligned}
& J_{i \ell} J_{j k, \ell}+J_{j \ell} J_{k i, \ell}+J_{k \ell} J_{i j, \ell} \\
& \quad=\left(H_{i}^{\mathrm{R}} m_{\ell}^{\mathrm{R}}-m_{i}^{\mathrm{R}} H_{\ell}^{\mathrm{R}}\right)\left(H_{j}^{\mathrm{R}} \delta_{k \ell}-\delta_{j \ell} H_{k}^{\mathrm{R}}\right) \\
& \quad+\left(H_{j}^{\mathrm{R}} m_{\ell}^{\mathrm{R}}-m_{j}^{\mathrm{R}} H_{\ell}^{\mathrm{R}}\right)\left(H_{k}^{\mathrm{R}} \delta_{i \ell}-\delta_{k \ell} H_{i}^{\mathrm{R}}\right) \\
& \quad+\left(H_{k}^{\mathrm{R}} m_{\ell}^{\mathrm{R}}-m_{k}^{\mathrm{R}} H_{\ell}^{\mathrm{R}}\right)\left(H_{i}^{\mathrm{R}} \delta_{j \ell}-\delta_{i \ell} H_{j}^{\mathrm{R}}\right) \\
& \quad=H_{i}^{\mathrm{R}} H_{j}^{\mathrm{R}} m_{k}^{\mathrm{R}}-H_{i}^{\mathrm{R}} H_{k}^{\mathrm{R}} m_{j}^{\mathrm{R}}-H_{j}^{\mathrm{R}} H_{k}^{\mathrm{R}} m_{i}^{\mathrm{R}}+H_{j}^{\mathrm{R}} H_{k}^{\mathrm{R}} m_{i}^{\mathrm{R}} \\
& \quad+H_{j}^{\mathrm{R}} H_{k}^{\mathrm{R}} m_{i}^{\mathrm{R}}-H_{i}^{\mathrm{R}} H_{j}^{\mathrm{R}} m_{k}^{\mathrm{R}}-H_{i}^{\mathrm{R}} H_{k}^{\mathrm{R}} m_{j}^{\mathrm{R}}+H_{i}^{\mathrm{R}} H_{k}^{\mathrm{R}} m_{j}^{\mathrm{R}} \\
& \quad+H_{i}^{\mathrm{R}} H_{k}^{\mathrm{R}} m_{j}^{\mathrm{R}}-H_{j}^{\mathrm{R}} H_{k}^{\mathrm{R}} m_{i}^{\mathrm{R}}-H_{i}^{\mathrm{R}} H_{j}^{\mathrm{R}} m_{k}^{\mathrm{R}}+H_{i}^{\mathrm{R}} H_{j}^{\mathrm{R}} m_{k}^{\mathrm{R}}=0 .
\end{aligned}
$$

Thus, $\mathbf{J}$ satisfies (9) and (10). Moreover, because $\mathbf{J}$ is a linear function of $\mathbf{m}^{\mathrm{R}}$, the bracket (8) with the above $\mathbf{J}$ is a Lie-Poisson bracket. Consequently, the Landau-Lifshitz equation (22) viewed in the corotated frame is a Lie-Poisson system (11).

As that presented in (12), from (69) we can identify the structure constants to be

$$
\begin{aligned}
C_{i j}^{1} & =\left[\begin{array}{ccc}
0 & -H_{2}^{\mathrm{R}} & -H_{3}^{\mathrm{R}} \\
H_{2}^{\mathrm{R}} & 0 & 0 \\
H_{3}^{\mathrm{R}} & 0 & 0
\end{array}\right], \\
C_{i j}^{2} & =\left[\begin{array}{ccc}
0 & H_{1}^{\mathrm{R}} & 0 \\
-H_{1}^{\mathrm{R}} & 0 & -H_{3}^{\mathrm{R}} \\
0 & H_{3}^{\mathrm{R}} & 0
\end{array}\right], \\
C_{i j}^{3} & =\left[\begin{array}{ccc}
0 & 0 & H_{1}^{\mathrm{R}} \\
0 & 0 & H_{2}^{\mathrm{R}} \\
-H_{1}^{\mathrm{R}} & -H_{2}^{\mathrm{R}} & 0
\end{array}\right] .
\end{aligned}
$$

Suppose that $\mathbf{m}^{\mathrm{R}}=m_{k}^{\mathrm{R}} \mathbf{e}_{k}$ and that $\left\{\mathbf{e}_{k}, k=1,2,3\right\}$ forms a basis of the dual Lie algebra $\mathcal{G}^{*}$. The above structure constants can be used to construct a Lie algebra denoted by $\mathcal{G}$ :

$$
\left[\mathbf{f}_{i}, \mathbf{f}_{j}\right]=C_{i j}^{k} \mathbf{f}_{k},
$$

where $\left\{\mathbf{f}_{k}, k=1,2,3\right\}$ forms a basis of the Lie algebra $\mathcal{G}$ and $[\bullet, \bullet]$ is the Lie commutator [40].

Next, we consider the adjoint representation of the Lie algebra $\mathcal{G}$. For each $\mathbf{f} \in \mathcal{G}$ the operator ad $\mathbf{f}$ that maps $\mathbf{g} \in \mathcal{G}$ into $[\mathbf{f}, \mathbf{g}]$ is a linear transformation of $\mathcal{G}$ onto itself, i.e.,

$$
(\operatorname{ad} \mathbf{f}) \mathbf{g}=[\mathbf{f}, \mathbf{g}]
$$

As supposed $\left\{\mathbf{f}_{k}, k=1,2,3\right\}$ is a basis for the Lie algebra $\mathcal{G}$ and then we have

$$
\left(\operatorname{ad} \mathbf{f}_{i}\right) \mathbf{f}_{j}=C_{i j}^{k} \mathbf{f}_{k} .
$$

Therefore the matrix associated with the transformation $\operatorname{ad} \mathbf{f}_{i}$ is

$$
\left(\mathbf{M}_{i}\right)_{j k}=C_{i k}^{j} .
$$

Corresponding to the structure constants given in (72)-(74), the following $\mathbf{M}_{i}$ are available:

$$
\mathbf{M}_{1}=\left[\begin{array}{ccc}
0 & -H_{2}^{\mathrm{R}} & -H_{3}^{\mathrm{R}} \\
0 & H_{1}^{\mathrm{R}} & 0 \\
0 & 0 & H_{1}^{\mathrm{R}}
\end{array}\right],
$$




$$
\begin{aligned}
& \mathbf{M}_{2}=\left[\begin{array}{ccc}
H_{2}^{\mathrm{R}} & 0 & 0 \\
-H_{1}^{\mathrm{R}} & 0 & -H_{3}^{\mathrm{R}} \\
0 & 0 & H_{2}^{\mathrm{R}}
\end{array}\right], \\
& \mathbf{M}_{3}=\left[\begin{array}{ccc}
H_{3}^{\mathrm{R}} & 0 & 0 \\
0 & H_{3}^{\mathrm{R}} & 0 \\
-H_{1}^{\mathrm{R}} & -H_{2}^{\mathrm{R}} & 0
\end{array}\right] .
\end{aligned}
$$

The above $\left\{\mathbf{M}_{k}, k=1,2,3\right\}$ indeed forms a matrix basis for the Lie algebra $\mathcal{G}$ and satisfies (75), because the Lie brackets of $\mathbf{M}_{k}, k=1,2,3$, satisfy

$$
\begin{aligned}
{\left[\mathbf{M}_{1}, \mathbf{M}_{2}\right] } & =\left[\begin{array}{ccc}
H_{1}^{\mathrm{R}} H_{2}^{\mathrm{R}} & \left(H_{2}^{\mathrm{R}}\right)^{2} & H_{2}^{\mathrm{R}} H_{3}^{\mathrm{R}} \\
-\left(H_{1}^{\mathrm{R}}\right)^{2} & -H_{1}^{\mathrm{R}} H_{2}^{\mathrm{R}} & -H_{1}^{\mathrm{R}} H_{3}^{\mathrm{R}} \\
0 & 0 & 0
\end{array}\right] \\
& =C_{12}^{1} \mathbf{M}_{1}+C_{12}^{2} \mathbf{M}_{2}, \\
{\left[\mathbf{M}_{1}, \mathbf{M}_{3}\right] } & =\left[\begin{array}{ccc}
H_{1}^{\mathrm{R}} H_{3}^{\mathrm{R}} & H_{2}^{\mathrm{R}} H_{3}^{\mathrm{R}} & \left(H_{3}^{\mathrm{R}}\right)^{2} \\
0 & 0 & 0 \\
-\left(H_{1}^{\mathrm{R}}\right)^{2} & -H_{1}^{\mathrm{R}} H_{2}^{\mathrm{R}} & -H_{1}^{\mathrm{R}} H_{3}^{\mathrm{R}}
\end{array}\right] \\
& =C_{13}^{1} \mathbf{M}_{1}+C_{13}^{3} \mathbf{M}_{3}, \\
{\left[\mathbf{M}_{2}, \mathbf{M}_{3}\right] } & =\left[\begin{array}{ccc}
0 & 0 & \\
H_{1}^{\mathrm{R}} H_{3}^{\mathrm{R}} & H_{2}^{\mathrm{R}} H_{3}^{\mathrm{R}} & \left(H_{3}^{\mathrm{R}}\right)^{2} \\
-H_{1}^{\mathrm{R}} H_{2}^{\mathrm{R}} & -\left(H_{2}^{\mathrm{R}}\right)^{2} & -H_{2}^{\mathrm{R}} H_{3}^{\mathrm{R}}
\end{array}\right] \\
& =C_{23}^{2} \mathbf{M}_{2}+C_{23}^{3} \mathbf{M}_{3} .
\end{aligned}
$$

$\mathbf{M}_{i}$ :

Let us consider the Lie group $\mathbf{G}_{i}$ generated from the matrix

$$
\dot{\mathbf{G}}_{i}=\mathbf{M}_{i} \mathbf{G}_{i}, \mathbf{G}_{i}(0)=\mathbf{I}_{3}, i \text { not summed }
$$

We can solve the above $\mathbf{G}_{i}$ :

$$
\begin{gathered}
\mathbf{G}_{1}=\left[\begin{array}{ccc}
1 & v_{12} & v_{13} \\
0 & \exp \left[\int_{0}^{t} H_{1}^{\mathrm{R}}(\eta) d \eta\right] & 0 \\
0 & 0 & \exp \left[\int_{0}^{t} H_{1}^{\mathrm{R}}(\eta) d \eta\right]
\end{array}\right], \\
\mathbf{G}_{2}=\left[\begin{array}{ccc}
\exp \left[\int_{0}^{t} H_{2}^{\mathrm{R}}(\eta) d \eta\right] & 0 & 0 \\
v_{21} & 1 & v_{23} \\
0 & 0 & \exp \left[\int_{0}^{t} H_{2}^{\mathrm{R}}(\eta) d \eta\right]
\end{array}\right],
\end{gathered}
$$

$$
\mathbf{G}_{3}=\left[\begin{array}{ccc}
\exp \left[\int_{0}^{t} H_{3}^{\mathrm{R}}(\eta) d \eta\right] & 0 & 0 \\
0 & \exp \left[\int_{0}^{t} H_{3}^{\mathrm{R}}(\eta) d \eta\right] & 0 \\
v_{31} & v_{32} & 1
\end{array}\right],
$$

where

$$
v_{i j}(t)=-\int_{0}^{t} H_{j}^{\mathrm{R}}(\xi) \exp \left[\int_{0}^{\xi} H_{i}^{\mathrm{R}}(\eta) d \eta\right] d \xi
$$

The above $\mathbf{G}_{i}$ is a dilational translation in the $i$-th plane $m_{i}^{\mathrm{R}}=$ constant denoted by $D T_{i}(2)$. The right-action of $D T_{i}(2)$ on $\mathbb{R}^{2}$ is a dilation followed by a translation with vector $\overline{\mathbf{v}}_{i}$ and has the following expression:

$$
\left(\overline{\mathbf{m}}_{i}^{\mathrm{R}}\right)^{\mathrm{T}}\left(e^{\int_{0}^{t} H_{i}^{\mathrm{R}}(\eta) d \eta} \mathbf{I}_{2}, \overline{\mathbf{v}}_{i}\right)=e^{\int_{0}^{t} H_{i}^{\mathrm{R}}(\eta) d \eta}\left(\overline{\mathbf{m}}_{i}^{\mathrm{R}}\right)^{\mathrm{T}}+\overline{\mathbf{v}}_{i}^{\mathrm{T}}
$$

with $\overline{\mathbf{m}}_{1}^{\mathrm{R}}=\left(m_{2}^{\mathrm{R}}, m_{3}^{\mathrm{R}}\right)^{\mathrm{T}}, \overline{\mathbf{m}}_{2}^{\mathrm{R}}=\left(m_{1}^{\mathrm{R}}, m_{3}^{\mathrm{R}}\right)^{\mathrm{T}}, \overline{\mathbf{m}}_{3}^{\mathrm{R}}=\left(m_{1}^{\mathrm{R}}, m_{2}^{\mathrm{R}}\right)^{\mathrm{T}} \in \mathbb{R}^{2}$, where $\overline{\mathbf{v}}_{1}=\left(v_{12}, v_{13}\right)^{\mathrm{T}}, \quad \overline{\mathbf{v}}_{2}=\left(v_{21}, v_{23}\right)^{\mathrm{T}}, \quad \overline{\mathbf{v}}_{3}=\left(v_{31}, v_{32}\right)^{\mathrm{T}} \in \mathbb{R}^{2}$. Note that we can embed $D T_{i}(2)$ into $G L(3, \mathbb{R})$ as in the formulation of (86)-(88), and thus one can operate with $D T_{i}$ (2) as one would with the matrix Lie groups by using the embedding technique.

Corresponding to the Lie algebra $\mathcal{G}$ there exists a Lie group denoted by $\mathbf{G}$ which is composed of all $D T_{i}(2)$, and the adjoint representation of the Lie group $\mathbf{G}$ is denoted by $\operatorname{Ad}_{\mathrm{g}}, \mathbf{g} \in \mathbf{G}$ :

$$
\operatorname{Ad}_{\mathrm{g}}: \mathcal{G} \mapsto \mathcal{G}
$$

$\mathcal{G}^{*}$ is foliated by the coadjoint orbits:

$$
\mathcal{O}_{\mathbf{m}^{\mathrm{R}}}=\left\{\operatorname{Ad}_{\mathrm{g}^{-1}}^{*} \mathbf{m}^{\mathrm{R}} \mid \mathbf{g} \in \mathbf{G}\right\} \subset \mathcal{G}^{*},
$$

where the coadjoint action $\mathrm{Ad}_{\mathrm{g}^{-1}}^{*}$ is defined by

$$
\left.<\operatorname{Ad}_{\mathrm{g}^{-1}}^{*} \mathbf{w}, \mathbf{v}>=<\mathbf{w}, \operatorname{Ad}_{\mathrm{g}^{-1}} \mathbf{v}\right\rangle, \mathbf{w} \in \mathcal{G}^{*}, \mathbf{v} \in \mathcal{G}
$$

Here $\langle\bullet, \bullet\rangle$ denotes a non-degenerate pairing between $\mathcal{G}^{*}$ and $\mathcal{G}$. For matrices the adjoint and coadjoint actions are, respectively, given by

$$
\operatorname{Ad}_{\mathbf{g}^{-1}} \mathbf{v}=\mathbf{g}^{-1} \mathbf{v g}
$$




$$
\operatorname{Ad}_{\mathbf{g}^{-1}}^{*} \mathbf{v}=\mathbf{g v}
$$

Deriving the pair in (93) with respect to $\mathbf{g}$ and then letting $\mathbf{g}$ equal to identity, we obtain

$$
<\operatorname{ad}_{\mathbf{u}}^{*} \mathbf{w}, \mathbf{v}>=<\mathbf{w}, \operatorname{ad}_{\mathbf{u}} \mathbf{v}>, \mathbf{w} \in \mathcal{G}^{*}, \mathbf{v} \in \mathcal{G}
$$

where $\mathbf{u}=\left.(d / d t) \mathbf{g}(t)\right|_{t=0} . \quad \mathrm{Ad}^{*}$ is the coadjoint representation of the Lie algebra $\mathcal{G}$. Then we have

$$
\operatorname{ad}_{\mathbf{f}_{i}}^{*} \mathbf{w}=-\mathbf{J}(\mathbf{w}) \mathbf{f}_{i}, \quad \mathbf{w} \in \mathcal{G}^{*}
$$

Therefore the matrix associated with the transformation $\operatorname{ad}_{\mathbf{f}_{i}}^{*}$ is

$$
\left(\mathbf{M}_{i}^{*}\right)_{j k}=-C_{j k}^{i}
$$

Since $C_{j k}^{i}$ is a skew-symmetric matrix for each $i$, the corresponding coadjoint action is found to be a three-dimensional rotation group denoted by $S O(3)$.

Given an initial point $\mathbf{m}^{\mathrm{R}}(0)$ on the invariant manifold, a solution to the Landau-Lifshitz equation (48) stays on the same coadjoint orbit $\mathcal{O}_{\mathbf{m}^{\mathrm{R}}(0)}$ for all time. Along the coadjoint orbit the generalized Hamiltonian function $H$ defined by (68) is a constant.

Observing (69) we can see that the rank of $\mathbf{J}$ is one when $\mathbf{m}^{\mathrm{R}}$ and $\mathbf{H}^{\mathrm{R}}$ are linearly dependent, and two when $\mathbf{m}^{\mathrm{R}}$ and $\mathbf{H}^{\mathrm{R}}$ are linearly independent. Because the rank of $\mathbf{J}$ is smaller than 3 , $\mathbf{J}$ is degenerate. For this case we have mentioned in Section 1 that there exists at least one Casimir function of the Landau-Lifshitz equation (48). However, this is not true for the Landau-Lifshitz equation. We demonstrate it below.

Suppose that there exists a function $C$, and its gradient is

$$
\nabla C=\left[\begin{array}{l}
\beta_{1} \\
\beta_{2} \\
\beta_{3}
\end{array}\right]
$$

with $\beta_{1}=m_{3}^{\mathrm{R}} H_{2}^{\mathrm{R}}-m_{2}^{\mathrm{R}} H_{3}^{\mathrm{R}}, \beta_{2}=m_{1}^{\mathrm{R}} H_{3}^{\mathrm{R}}-m_{3}^{\mathrm{R}} H_{1}^{\mathrm{R}}, \quad \beta_{3}=m_{2}^{\mathrm{R}} H_{1}^{\mathrm{R}}-$ $m_{1}^{\mathrm{R}} H_{2}^{\mathrm{R}}$. It is easy to prove that

$$
\left[\begin{array}{l}
\beta_{1} \\
\beta_{2} \\
\beta_{3}
\end{array}\right]^{\mathrm{T}}\left[\begin{array}{ccc}
0 & \beta_{3} & -\beta_{2} \\
-\beta_{3} & 0 & \beta_{1} \\
\beta_{2} & -\beta_{1} & 0
\end{array}\right]=\left[\begin{array}{l}
0 \\
0 \\
0
\end{array}\right]^{\mathrm{T}}
$$

Therefore, the Lie-Poisson bracket of such $C$ with any differentiable function $F$ is zero, that is, $\{C, F\}=0$. By definition if such $C$ exists it seems a Casimir function. However, Eq. (99) can be expressed as

$$
\nabla C=\left[\begin{array}{ccc}
0 & -H_{3}^{\mathrm{R}} & H_{2}^{\mathrm{R}} \\
H_{3}^{\mathrm{R}} & 0 & -H_{1}^{\mathrm{R}} \\
-H_{2}^{\mathrm{R}} & H_{1}^{\mathrm{R}} & 0
\end{array}\right]\left[\begin{array}{l}
m_{1}^{\mathrm{R}} \\
m_{2}^{\mathrm{R}} \\
m_{3}^{\mathrm{R}}
\end{array}\right]
$$

and we can see that it is impossible to have a function that its gradient is the multiplication of a skew-symmetric matrix with the vector $\mathbf{m}^{\mathrm{R}}=\left(m_{1}^{\mathrm{R}}, m_{2}^{\mathrm{R}}, m_{2}^{\mathrm{R}}\right)^{\mathrm{T}}$. Thus, we conclude that for the Landau-Lifshitz equation (22) there has no Casimir function even the rank of $\mathbf{J}$ is smaller than the dimensions 3 .

The above statement seems to contradict to the statement about the Lie-Poisson system. However, the premise which this statement holds is that the state point $\mathbf{x}$ is a regular point. But for the Landau-Lifshitz equation the state point $\mathbf{m}^{\mathrm{R}}$ is a singular point in the manifold.

\section{CONCLUSION}

In this paper we have investigated the Landau-Lifshitz equation from several theoretical aspects. We have explored six types of representations for the Landau-Lifshitz equation. The six representations are compared in Table 1 by considering their underlying space, bracket structure, generator, invariance, Lie algebra, and Lie group. Each has its philosophy as being a different aspect of the same magnetization behavior of ferromagnetic materials modeled by the Landau-Lifshitz equation. In the six representations only the linear system formulation does not rely on the use of bracket, while the other five representations have used one or two of the following brackets: Lie, Lie-Poisson, Poisson, dissipative, and double.

We proved that the two-generator formalism nicely highlights the conservative magnetization and magnetic hysteretic behavior of the Landau-Lifshitz equation. By mapping it into the skew-symmetric matrix space we also developed an isospectral flow model as well as a double-bracket flow model of the Landau-Lifshitz equation. Then, we have explored the internal symmetry group inherent in the Landau-Lifshitz equation, of which the proper orthochronous Lorentz group $\mathrm{SO}_{o}(3$, 1) was found to be an underlying symmetry group.

In terms of the generalized Hamiltonian formalism we can explore the invariant behavior of conservative magnetization magnitude as a coadjoint orbit on a symplectic foliation in the dual Lie algebra space. The invariant function plays the role of a generalized Hamiltonian function in the Lie-Poisson system. Conversely, in the dissipative bracket formulation the hysteretic behavior of ferromagnetic material is reflected in the magnetic dissipation as a generator. The invariant behavior is guaranteed by preserving the Casimir function invariant. While the Lie-Poisson system formulation stresses only the invariant behavior through the coadjoint orbit, both the two-generator and linear system formulations make a further breakthrough of the concept by protruding the dissipation nature of magnetic hystereticity and keeping the magnetization orbit in the invariant manifold. In the six types of 
representations, the one with the linear system representation seems the best. Even disregarding its linearity, the advantages of this formulation are reflected by its simplicity on the future pointing of $X^{0}$ due to the magnetic dissipation and the retention of the invariant manifold by the cone in the fourdimensional Minkowski space.

\section{ACKNOWLEDGMENTS}

The financial supports provided by the National Science Council for CS under the Grant NSC 93-2212-E-019-008 and for KC under NSC 97-2221-E-002-12 are gratefully acknowledged.

\section{REFERENCES}

1. Beris, A. N., "Bracket formulation as a source for the development of dynamic equations in continuum mechanics," Journal of Non-Newtonian Fluid Mechanics, Vol. 96, pp. 119-136 (2001).

2. Bertotti, G., Serpico, C., and Mayergoyz, I. D., "Nonlinear magnetization dynamics under circularly polarized field," Physical Review Letters, Vol. 86, pp. 724-727 (2001)

3. Bloch, A., Krishnaprasad, P. S., Marsden, J. E., and Ratiu, T. S., "The Euler-Poincaré equations and double bracket dissipation," Communications in Mathematical Physics, Vol. 175, pp. 1-42 (1996).

4. Brockett, R. W., "Dynamical systems that sort lists, diagonalize matrices, and solve linear programming problems," Linear Algebra and its Applications, Vol. 146, pp. 79-91 (1991).

5. Channell, P. J. and Scovel, J. C., "Integrators for Lie-Poisson dynamical systems," Physica D, Vol. 50, pp. 80-88 (1991).

6. Chen, K. C., "Noncanonical Poisson brackets for elastic and micromorphic solids," International Journal of Solids and Structures, Vol. 44, pp. 7715-7730 (2007).

7. Chen, K. C., "Constructing Poisson and dissipative brackets of mixtures by using Lagrangian-to-Eulerian transformation," Journal of Mechanics, (2009) (in press).

8. Chu, M. T. and Driessel, K. R., "The projected gradient method for least squares matrix approximations with spectral constraints," SIAM Journal of Numerical Analysis, Vol. 27, pp. 1050-1060 (1990).

9. De Pablo, J. J. and Öttinger, H. C., "An atomistic approach to general equation for the nonequilibrium reversible-irreversible coupling," Journal of Non-Newtonian Fluid Mechanics, Vol. 96, pp. 137-162 (2001).

10. EngØ, K. and Faltinsen, S., "Numerical integration of Lie-Poisson systems while preserving coadjoint orbits and energy," SIAM Journal of Numerical Analysis, Vol. 39, pp. 128-145 (2001).

11. Fetisov, Y. K., Patton, C. E., and Sygonach, V. T., "Nonlinear ferromagnetic resonance and foldover in yttrium iron garnet thin films-inadequacy of the classical model." IEEE Transaction on Magnetics, Vol. 35, pp. 4511-4521 (1999).

12. Frank, J., "Geometric space-time integration of ferromagnetic materials,' Applied Numerical Mathematics, Vol. 48, pp. 307-322 (2004).

13. Ge, Z. and Marsden, J. E., "Lie-Poisson Hamiltonian Jacobi theory and Lie-Poisson integrator,” Physics Letters A, Vol. 133, pp. 134-139 (1988).

14. Gilbert, T. L., "A Lagrangian formulation of the gyromagnetic equation for the magnetic field," Physical Review, Vol. 100, p. 1243 (1955).

15. Grmela, M., "Bracket formulation of dissipative fluid mechanics equations," Physics Letters A, Vol. 102, pp. 355-358 (1984).

16. Grmela, M. and Öttinger, H. C., "Dynamics and thermodynamics of complex fluids: I. development of a general formalism," Physical Review E, Vol. 56, pp. 6620-6632 (1997).

17. Iserles, A., "On the discretization of double-bracket flows," Foundations in Computational Mathematics, Vol. 2, pp. 305-329 (2002).
18. Kaufman, A. N., "Dissipative Hamiltonian systems: a unifying principle," Physics Letters A, Vol. 100, pp. 419-422 (1984).

19. Krishnaprasad, P. S. and Tan, X., "Cayley transformations in micromagnetics," Physica B, Vol. 306, pp. 195-199 (2001).

20. Landau, L. D. and Lifshitz, E. M., "On the theory of the dispersion of magnetic permeability in ferromagnetic bodies," Physik. Z. Soviet Union, Vol. 8, pp. 153-169 (1935).

21. Li, S. T. and Qin, M., "Lie-Poisson integration for rigid body dynamics," Computers \& Mathematics with Applications, Vol. 30, pp. 105-118 (1995).

22. Liu, C.-S., "A Jordan algebra and dynamic system with associator as vector filed," International Journal of Non-Linear Mechanics, Vol. 35, pp. 421-429 (2000).

23. Liu, C.-S., "Cone of non-linear dynamical system and group preserving schemes," International Journal of Non-Linear Mechanics, Vol. 36, pp. 1047-1068 (2001).

24. Liu, C.-S., "Applications of the Jordan and Lie algebras for some dynamical systems having internal symmetries," International Journal of Applied Mathematics, Vol. 8, pp. 209-240 (2002).

25. Liu, C.-S., "Lie symmetry of the Landau-Lifshitz-Gilbert equation and exact linearization in the Minkowski space," Zeitschrift für angewandte Mathematik und Physik (ZAMP), Vol. 55, pp. 606-625 (2004).

26. Liu, C.-S., "Five different formulations of the finite strain perfectly plastic equations," CMES: Computer Modeling in Engineering \& Sciences, Vol. 17 pp. 73-93 (2007).

27. Liu, C.-S., "The computation of modified Landau-Lifshitz equation under an AC field," CMC: Computers, Materials, \& Continua, Vol. 5, pp. 151-159 (2007).

28. Liu, C.-S., "A consistent computation of magnetization reversal under a circularly polarized field and anisotropy field," CMC: Computers, Materials, \& Continua, Vol. 6, pp. 117-128 (2007).

29. Liu, C.-S. and Ku, Y.-L., "A combination of group preserving scheme and Runge-Kutta method for the integration of Landau-Lifshitz equation," CMES: Computer Modeling in Engineering \& Sciences, Vol. 9, pp. 151-178 (2005).

30. Marsden, J. E. and Ratiu, R. S., Introduction to Mechanics and Symmetry, Springer-Verlag, New York (1994).

31. McLachlan, R. I., "Explicit Lie-Poisson integration and the Euler equations," Physical Review Letters, Vol. 71, pp. 3043-3046 (1993).

32. Morrison, P. J., "Bracket formulation for irreversible classical fields," Physics Letters A, Vol. 100, pp. 423-427 (1984).

33. Öttinger, H. C., "GENERIC projection-operator derivation of Boltzmann's kinetic equation," Journal of Non-Equilibrium Thermodynamics, Vol. 27, pp. 105-119 (2002)

34. Öttinger, H. C. and Grmela, M., "Dynamics and thermodynamics of complex fluids: II. illustrations of a general formalism," Physical Review E, Vol. 56, pp. 6633-6655 (1997).

35. Pelino, V. and Pasini, A., "Dissipation in Lie-Poisson systems and the Lorenz-84 model," Physics Letters A, Vol. 291, pp. 389-396 (2001).

36. Safonov, V. L., "Tensor form of magnetization damping," Journal of Applied Physics, Vol. 91, pp. 8653-8655 (2002).

37. Safonov, V. L. and Bertram, H. N., "Nonlinear microscopic relaxation of uniform magnetization precession," Journal of Applied Physics, Vol. 93, pp. 6912-6914 (2003).

38. Schrefl, T., Fidler, J., Süss, D., and Scholz, W., "Hysteresis and switching dynamics of patterned magnetic elements," Physica B, Vol. 275, pp. 55-58 (2000).

39. Serpico, C., Mayergoyz, I. D., and Bertotti, G., "Numerical technique for integration of the Landau-Lifshitz equation," Journal of Applied Physics, Vol. 89, pp. 6991-6993 (2001).

40. Varadarajan, V. S., Lie Groups, Lie Algebras, and Their Representations, Springer-Verlag, New York (1984).

41. Zhai, J., "Dynamics of domain walls in ferromagnets and weak ferromagnets," Physics Letters A, Vol. 234, pp. 488-492 (1997). 